\title{
Object Unified Identifier Method in Logistics Resource Integration
}

\author{
Huiqi Zhu, Hanping Hou \\ Beïing Jiaotong University (China) \\ zbubuiqi0505@126.com,_boubanping@126.com
}

Received: December 2014

Accepted: February 2015

\section{Abstract:}

Purpose: The status which many programs for the object identifier are not incompatible with each other has become a bottleneck for integrating logistics resources. Scholars have done some relevant studies in terms of coding and conversion mechanisms, but the problem still has not got a better solution. The purpose of this study is to research how to make the object identifier programs compatible.

Design/methodology/approach: Author proposed an object unified identifier (OUID) method based on OID and introduced the standard identification code in it, according to the problems of the object identifier in logistics resource integration. And the paper further designed the acquisition process of the resource basic information and location information, and analyzed the application environment of object unified identifier based on OID.

Findings: OUID made up for the lack of location information in conversion mechanism, and avoided to promote new unified identifier standards at the same time. The supplement of the application environment provided an important support to solve the problems of poor communication caused by non-unified object identifier in the process of logistics resource integration.

Originality/value: Using this method, each identification system not only can keep its own territory, but also can compatible with other object identifiers.

Keywords: logistics resource integration; object identifier; object unified identifier 


\section{Introduction}

In recent years, Internet of things (IOT) and other IT technologies enable logistics resources to connect with the Internet through information devices, which let the logistics resources have the connectivity, visibility and virtuality and give the resources ideas. It is helpful to realize intelligent identification and management and provides a new way of thinking for the logistics resource integration. But the inconsistent resource identifier, the equipment that access information relied on belonging to different organizations, greatly reduced the effectiveness and accuracy of the logistics resource search (Yu \&Yu, 2013), and restricted the improvement of logistics resource integration. Especially the heterogeneity and high dispersion of data sources under the Internet of things (Hribernik, Kramer, Hans \& Thoben, 2010), made uniquely identifying and positioning logistics resource more difficult. When enterprises intended to complete cross-domain integration of logistics resources, they felt that it was difficult to manage and operate information in a unified way in network. Such as manufacturing enterprises used the bar-code to identify the products or pallets, but when the products or pallets got into the logistics field, logistics enterprises didn't use the identifier continuously, and adopt their own identifiers and the rules. This phenomenon led to the same resource having different identifiers in different logistics information systems, caused the inconsistency of object identifiers, and made resource information scattered and logistics resources operating uncoordinated. Thus, an object unified identifier (OUID) becomes the key issue in integrating logistics resources.

Pålsson and Johansson (2009) found that the unified identifier of goods had positive impact on supply chain integration through researching Swedish manufacturing companies. This study made people realized important role of the unique identifier for integration. If all the logistics resources have the unified identifiers, it will easy to ensure the reliability, security, interoperability of the resource information in the logistics process (Ilie-Zudor, Kemény, van Blommestein, Monostori \& van der Meulen, 2011), and to support the dynamic integration and seamless links of logistics resources (Prajogo \& Olhager, 2012; Romano, 2003). There are several different programs for the object identifier according to the applications, CASAGRAS report (2010) analyzed the world's major object identifier systems in detail, but it had not yet been expounded how to form a unified identifier program. Due to the existence of a large number of heterogeneous history data, promoting the application of unified identifier standard was impractical. Therefore, the research emphasis of the object identifier should be placed in the global unified compatible identifier system (CERP-IOT, 2009). Current unified identifier schemes are mainly focus on the information coding and addressing. In terms of coding, the researchers placed extra emphasis on compiling new code (Yang, 2013). Some of them (Yang, 2013; Sun, Zhang, Qin, Tu, Wang \& Wu, 2013) added the location information into the new code to meet the IoT demand for it. However, it was not benefit to achieve unification, because new code would aggravate the heterogeneous status of identifiers. In the IoT addressing, research mainly focused on the new IoT networking architecture (Karakostas, 2013; Liu, Yan, 
Tian, Kong, Li \& Yan, 2014) or conversion mechanism (Kong, 2008; Pang, 2012; Jung, Choi, Lee $\&$ Kim, 2012) in order to find the existing information in network. Some scholars discussed the problem from the perspective of computer languages. For example, Martinez-Julia and Skarmeta (2013) believed that the future Internet was a mobile, host-free node model with the possibility of multiple attachment points to the network, and needed flexible naming and integrated discovery mechanisms. They tried to decouple the identification and location (Spleiß \& Kunzmann, 2011) to adapt to the trend of dynamic networks. But scholars in the field of USN thought IP addresses were too long to fit on small embedded devices. These studies provided the new perspective for the unified object identifier, but it was difficult to be realized because of lower operability. Therefore, according to the standardized method, firstly, this study applied conversion thought to research how to make the existing object identifier unified using the OID standard, and at the same time ensured that no new coding standard was generated. Secondly, relying on the Internet discovery mechanism, compatible and operable network architecture was proposed which can achieve the unifying of the object identifier.

\section{Object Identifier Basic Philosophy in Logistics Resource Integration}

The identifier mentioned in this article referred to the sign or symbol that showed the properties of an object in the form of a physical or electronic data. If the resources have the identifiers, logistics bodies can identify them, and then unique find the related information in network. This was also the main task of the object identifiers undertook in the process of logistics resource integration. Logistics resources mainly referred to the infrastructure and ancillary equipment that scattered in various functional resources for completing the logistics missions, for instance, shelves, trays, transport vehicles, positioning equipment, goods, etc.

\subsection{Object Identifier Operation Conceptual Model}

The operating process of the object identifier was described by a conceptual model in this section, as shown in Figure 1. It contained four parts, such as coding, labeling, identifying, and decoding.

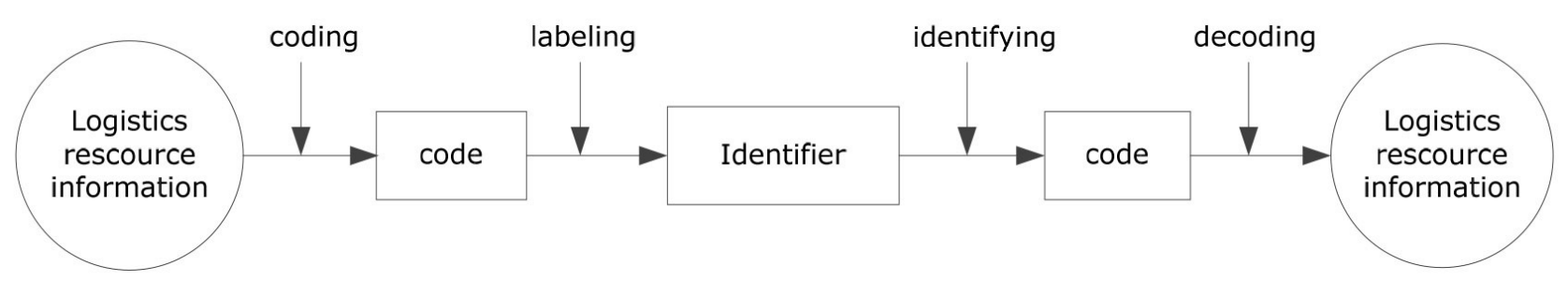

Figure 1. Object Identifier Operation Conceptual Model (Zhang, 2012) 
When the logistics resource information needed to be collected automatically, first of all, it should be coded and then formed codes that could be identified by a computer. Encoding process of the codes needed to follow certain standards, and requiring the code could indicate only one object. After generating the code, it must be combined with the resource, otherwise, it was a meaningless symbol. The purpose of the labeling was to link the resources with the codes that had been written, and formed the resources unified identifiers. When logistics resources participated in the social and economic activities, people could only clear the state of the logistics resources by identifying the identifiers in order to provide assistance for controlling, managing and making integrated decision. To improve the utilization rate of resources, enterprises needed to timely understand itself and resources state in society. For this reason, they required identified the resources. There were lots of technologies to identify the identifiers, and all of them were to obtain the loaded codes in the identifiers to receive the resource information. Decoding was the process of restoring the resource information that included the encapsulation, unpacking, resolving, etc., which was mainly completed by computers. It should be noted that decoding might not be completed in the network because of the non-unified object identifier of the logistics resources. This was also the core problem that was solved in this study.

\subsection{Problems of the Object Identifier}

From the object identifier operation conceptual model, you can see that the main role of the object identifier is to let the logistics resources be connected with network so that logistics bodies can control and manage the resources through the internet. But the status of the numerous objects identification technologies led that identifier could only be identified within the scope of its corresponding system. This made realizing the above function having difficulties, and some problems had been reflected as follows,

1. There was large difference of coding standards, so the expression of the same information faced the problem of choosing different standards. For example, Ucode and mRFID Code both have a 128-bit long code, but they have different structures. EPC has various classes of codes, such as EPC-64, EPC-256, and so on. And each category can be further subdivided. Therefore, coding standards difference caused the coding inconsistent phenomenon in the expression of resource information.

2. Each standard was applicable to a limited extent. As in the application of RFID technology, ISO 17363 and ISO 17364 were the coding standards of logistics containers, and ISO 15961 was for objects that were identified by RFID. In addition, in the EPCglobal standards system belonged to GS1 also had standards for objects or logistics units. Thus, the standards were only applicable to a limited extent. 
3. The same resource information obtained through different collection means, and stored in databases of different bodies. Some information could be traced and inquired in local area by technologies such as EPC, but most information was also still in the vertical closed-loop applications system and couldn't communicate with each other. Lead to resource information was scattered and not manageable.

\subsection{The Demand for OUID}

The problems existing in the object identifier mentioned above was manifested mainly in field of logistics. Especially, when the enterprise needed a lot of accurate and timely cross-domain resource information (including resources basic information and location information) to integrate the logistics resources, low search success rate and accuracy problems of logistics resources became more prominent brought with non-unified object identifier. Logistics section of supply chain operation reference model (SCOR) reflected the application status of the objects identification technology in the logistics resources. Analyzing the SCOR 10.0 can also get the logistics resource information that need collected in the logistics process (as shown in Table 1). This information was the basis of integrating logistics resources.

From the Table 1, we saw that a lot of identification technologies could be adopted by logistics resource information. Some resource information, such as transportation route, vehicle information, temporary location, didn't need be labeled on the resource, but they need be found by the identifiers. When the process of integration need them, they could not be received because the different identifiers used by different nodal enterprises. The phenomenon that the information was scattered in different database of the nodal enterprises was very obvious. So, in order to improve the efficiency of the logistics resources integration, the unified object identifiers was an essential ring. Moreover, non-unified identifiers issue had caused the attention of society. The national development and reform commission set up the national IoT based standard working group (NBSWG) in November 2010, National IoT coding identification project team (NCIPT) was one of the three groups which made up of the NBSWG. NCIPT's the key mission was to solved the problems of logistics resource information expression caused by chaotic application environment of object identifiers. To reduce the impact on logistics resource integration, this paper will perfect the object identifier from the following aspects. 


\begin{tabular}{|c|c|c|c|c|}
\hline $\begin{array}{l}\text { Logistics } \\
\text { links }\end{array}$ & Process code & Process name & $\begin{array}{l}\text { Resource Information to be } \\
\text { collected }\end{array}$ & $\begin{array}{l}\text { The main } \\
\text { identification } \\
\text { technology }\end{array}$ \\
\hline Source & $\begin{array}{l}(\mathrm{sS} 1.2 / \mathrm{sS} 2.2 / \mathrm{sS} 3.4) / \\
(\mathrm{sS} 1.3 / \mathrm{sS} 2.3 / \mathrm{sS} 3.5) / \\
(\mathrm{sS} 1.4 / \mathrm{sS} 2.4 / \mathrm{sS} 3.6)\end{array}$ & $\begin{array}{l}\text { Receive, verify, } \\
\text { transfer product }\end{array}$ & $\begin{array}{c}\text { Product information (name, } \\
\text { specification, quality, quantity) } \\
\text { Enterprise information (vendor } \\
\text { name) } \\
\text { Transportation Information } \\
\text { (vehicle information) } \\
\text { Storage Information (the } \\
\text { shelves number information) }\end{array}$ & $\begin{array}{l}\text { Bar-code, RFID, } \\
\text { Enterprise } \\
\text { independent coding } \\
\text { techniques }\end{array}$ \\
\hline \multirow{4}{*}{ Make } & sM1.3/sM2.3/sM3.4 & Produce and Test & \multirow{4}{*}{$\begin{array}{c}\text { Product information (machining } \\
\text { process, quality, package, test) } \\
\text { Storage Information (temporary } \\
\text { location) }\end{array}$} & \multirow{4}{*}{$\begin{array}{l}\text { Bar-code, RFID, } \\
\text { Enterprise } \\
\text { independent coding } \\
\text { techniques }\end{array}$} \\
\hline & sM1.4/sM2.4/sM3.5 & Package & & \\
\hline & sM1.5/sM2.5/sM3.6 & Stage Product & & \\
\hline & sM1.6/sM2.6/sM3.7 & $\begin{array}{l}\text { Release Finished } \\
\text { Product to Deliver }\end{array}$ & & \\
\hline \multirow{6}{*}{ Deliver } & sD1.8/sD2.8/sD3.8 & $\begin{array}{l}\text { Receive Product } \\
\text { from Source or } \\
\text { Make }\end{array}$ & \multirow{6}{*}{$\begin{array}{l}\text { Product information (name, } \\
\text { specification, quantity) } \\
\text { Enterprise information } \\
\text { (enterprise name and address) } \\
\text { Transportation Information } \\
\text { (logistics service providers, } \\
\text { vehicle information, container } \\
\text { information, transportation } \\
\text { route) } \\
\text { Storage Information (inflow } \\
\text { record and outflow record, the } \\
\text { shelves number information) }\end{array}$} & \multirow{6}{*}{$\begin{array}{c}\text { Bar-code, RFID, } \\
\text { GPS, Enterprise } \\
\text { independent coding } \\
\text { techniques, Sensor } \\
\text { technology }\end{array}$} \\
\hline & sD1.9/sD2.9/sD3.9 & Pick Product & & \\
\hline & sD1.10/sD2.10/sD3.10 & Pack Product & & \\
\hline & sD1.11/sD2.11/sD3.11 & $\begin{array}{c}\text { Load Vehicle \& } \\
\text { Generate Shipping } \\
\text { Docs }\end{array}$ & & \\
\hline & sD1.12/sD2.12/sD3.12 & Ship Product & & \\
\hline & sD1.13/sD2.13/sD3.13 & $\begin{array}{l}\text { Receive and Verify } \\
\text { Product by } \\
\text { Customer }\end{array}$ & & \\
\hline \multirow{3}{*}{ Return } & sDR1.3/sDR1.4/sSR1.5 & $\begin{array}{l}\text { Receive/transfer/r } \\
\text { eturn Defective } \\
\text { Product (includes } \\
\text { verify) }\end{array}$ & \multirow{3}{*}{$\begin{array}{l}\text { Product information (name, } \\
\text { specification, quantity) } \\
\text { Transportation Information } \\
\text { (Logistics service providers, } \\
\text { vehicle information, return and } \\
\text { exchange goods transportation } \\
\text { route) }\end{array}$} & \multirow{3}{*}{$\begin{array}{l}\text { Bar-code, RFID, } \\
\text { GPS, Enterprise } \\
\text { independent coding } \\
\text { techniques }\end{array}$} \\
\hline & sDR2.3/sDR2.4/sSR2.5 & $\begin{array}{l}\text { Receive/Transfer / } \\
\text { return MRO } \\
\text { Product (includes } \\
\text { verify) }\end{array}$ & & \\
\hline & sDR3.3/sDR3.4/sSR3.5 & $\begin{array}{l}\text { Receive/Transfer / } \\
\text { return Excess } \\
\text { Product }\end{array}$ & & \\
\hline
\end{tabular}

Table 1. Sub-processes of Using Identification Technology in SCOR10.0 Model

(1) Unique. The object identifier must be unique in the world, and it can be the temporary identifier of resource (Spleiß \& Kunzmann, 2011).

(2) Compatibility. Under the premise of unchanging existed identifier standard, it can achieve finding and transforming among the various object identifier information.

(3) Scalability. When new requirements or new coding scheme emerged, object identifier can add fields to express meaning accordingly.

(4) Manageability. Allow administrators to control data administrator, to realize the traceability of logistics information, classifying information, monitoring and management information, dynamic routing forwarding, etc. 


\section{Method of Logistics Resource Integration OUID}

Almost all standardization organization declared its standardization structure can be compatible with other standards, but different standard compatible problems remain existed. Analyzing the reasons that the heterogeneity problems of object identifier weren't solved nicely, we can find the following arguments. Firstly, a large number of heterogeneous and valuable historical data existed in network and enterprise database with the development of identification technologies. Secondly, internet information addressing mechanism did not adapt to the development of the IoT. Lastly, organizational interests hindered the unified of identifier. So promoting and applying the unified identifier standards was unrealistic, we needed to discuss the OUID method on the basis of unchanging the existed standards.

\subsection{Problem Description}

Logistics resource integration can achieve intelligent identification of resource, and knowing the location of logistics resources through the object identification technology under the IoT environment. But the current logistics system was designed based on internet host information discovery mechanism, so logistics resource unified identifier shouldn't be separated from internet application condition. Therefore, Logistics resources OUID should meet the requirements of the internet, and at the same time adapt to the rapid development of IoT. Then there would be considered the internet reality condition and physical location information. The following assumptions have been proposed.

(1) OUID mainly used the existing severs, and relied on internet information discovery mechanism.

(2) Logistics resources integration required the identification information included the resource basic information and location information. Resource basic information referred to the information that could reflect the nature of resources mostly and had the characteristics of shared demands across business systems. Location information referred to the physical location of logistics resources.

(3) Have designed specialized hardware devices which could input the entity encoding information into tags and computers.

(4) Logistics bodies generally accepted and applied the scheme of unified identifier.

Under the above assumptions, there will be help to realize the integration of logistics resources through perfecting the unified identifiers. 


\subsection{The Selection of the OUID Method}

Compared coding structures of EPC, UID, MCODE, and CPC, which have been widely applied (as shown in Table 2), we can find that existing standard coding structure generally was consisted of organization or enterprise code information, coding structure information, and object categories information. Although some of the resource information has already been contained in object identifier, more detailed information which will be available by further addressing, such as product quality, logistics service providers, inflow and outflow record, the shelves number information, still stored in the relevant enterprises databases or organizations databases. In terms of location information, the physical location information of resources was commonly out of the coding structure scope. Although location codes can be accomplished by the EPC and UID (as shown in Table 2), these location codes cannot be directly associated with encoded resources. Analysis also found that almost all of the identifier codes had the hierarchical structure, but it could not be directly identified from the codes. When the different identifier codes appeared, people had no way to know the expressed information of the codes, and that which identification standard system the codes belonged to. Therefore, EPC, UID, MCODE, CPC were all not suitable as a unified method.

Compared with the above four kinds of coding structure, Ecode achieved compatibility among the codes mainly by the way of distributing header on the basis of keeping the original coding structure. It was much more to provide compatible coding solutions. This study did not choose Ecode as the method of unified identifiers, because it was developed by our own country and its universality in the international arena was lower, furthermore, its coding structure was fixed that was not conducive to extend the resource information.

\begin{tabular}{|c|c|c|c|c|c|c|}
\hline $\begin{array}{l}\text { Identification } \\
\text { standards program }\end{array}$ & $\begin{array}{c}\text { Country, } \\
\text { Organization or } \\
\text { company code }\end{array}$ & $\begin{array}{l}\text { Coding } \\
\text { structure }\end{array}$ & $\begin{array}{l}\text { Object } \\
\text { category }\end{array}$ & $\begin{array}{c}\text { Object } \\
\text { description }\end{array}$ & $\begin{array}{c}\text { Whether having } \\
\text { the location code } \\
\text { or not }\end{array}$ & $\begin{array}{l}\text { Identifier } \\
\text { standards } \\
\text { code }\end{array}$ \\
\hline EPC & $\sqrt{a}$ & $\sqrt{ }$ & $\sqrt{ }$ & $\sqrt{ }$ & $\sqrt{ }$ & \\
\hline UID & $\sqrt{ }$ & $\sqrt{ }$ & $\sqrt{ }$ & & $\sqrt{ }$ & \\
\hline CPC & $\sqrt{ }$ & $\sqrt{ }$ & $\sqrt{ }$ & $\sqrt{ }$ & & \\
\hline MCODE & $\sqrt{ }$ & $\sqrt{ }$ & $\sqrt{ }$ & & & \\
\hline ECODE & $\sqrt{ }$ & $\sqrt{ }$ & $\sqrt{ }$ & $\sqrt{ }$ & $\sqrt{ }$ & $\sqrt{ }$ \\
\hline
\end{tabular}

${ }^{\mathrm{a}} \sqrt{ }$ represents the information contained

Table 2. Comparison Table of Objects Code and Structure

The OID was an identification system defined by ISO/IEC 8824 and ISO/IEC 9834 series standards. Using the OID code as prefix for all kinds of object identifier code, which will ease incompatible problem of identifier codes, can realize be compatible with object identifiers supported by any standard and the resolving of object identifier coding structure. Especially 
difficulty of using OID to convert identifiers was very low, it was easy to maintain and guarantee good expansibility. OID coding structure was a hierarchical authorization, so there was conducive to the interconnection of countries. In addition, OID supported people read and machine read, and could be converted into binary and be stored in RFID, sensor, two dimensional bar-code and other carriers. In this paper, the unified method of object identifiers will be mainly use identifier code based on OID to complete the compatibility and query between the identifier information.

In order to confirm logistics resource type, quantity, function, capability and feasible mode of transportation among the various facilities in a certain space (Shan, Zhang, Wang \& Chen, 2011), resource location information was needed except for the basic information. It was explained by analyzing OUID application environment that utilized the existing network topology.

\subsection{OUID Method Based on OID}

Structure of the OUID was standard identification code + resource code. The standard identification code was used to uniquely express coding standard code that object identifier code belongs to, and its allocation rules followed OID standard (GB/T 26231-2010, 2010). The allocation management of the standard identification code was shown in Figure 1. Allocating standard identification code based on the OID standard can transform different coding structures into the unified grading ones.

For instance, An Ecode-64 logistics resources code can get standard identification code just like 1.2.156.x.x.x ( $x$ indicated a set of indefinite length code). Resource code can be allocated according to the standard of different identification system. GS1 applied for 2.51 as identifiers prefix which was used to manage resource objects in the logistics field. If a logistics resource object's bar code was 6937512503343, its standard identification code can be 2.51.x.x. After being unified, logistics resource object identifier will become 2.51.x.x.6937512503343. Object identifier coding standard with the unique standard identification code can help complete decoding the object identifier code. As can be seen from the sample, allocation right of standard identification code belonged to identifier standards management department. Distributed management helped to realize countries and standard organizations manage coding standard, code type dispersedly and independently, and avoided interest conflict that caused by centralized management of identifier standard (Jung, et al., 2012).

The current network structure can't resolve the OUID based on OID. For this reason, the next section will analyze the network application environment and reveal the compatibility of the OUID on the basis of the existing internet discovery mechanism. 


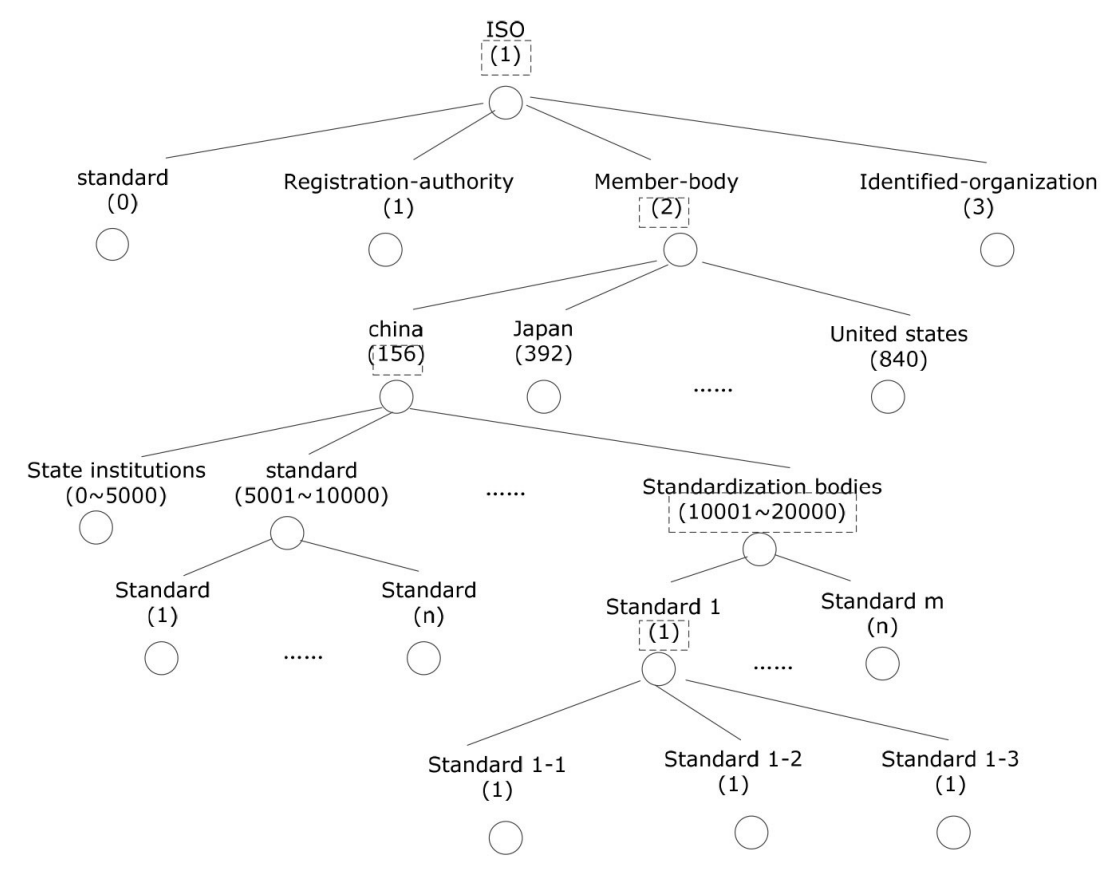

Figure 2. Application Example of Standard Identification Code Allocation

\section{Application of Logistics Resource Integration OUID}

\subsection{Application Environment}

To make OUID based on OID give full play to the role in the network, there needed to resolve the identifier so as to complete acquisition of resource detailed information. However, the network cannot do the job. To solve the problem, we introduced the identifier resolution layer (IRL), shown in Figure 3.

IRL firstly resolved OUID, and then decided whether through the DNS to obtain complete information according to the resolution results. So IRL was the network structure composed by many servers, included DNS resolution servers, OID servers and other standard organization servers, etc. (as shown in Figure 4).

When computers received a query of the object identifier, it was sent directly to IRL if it was OUID, otherwise it needed to be packaged in wrappers. OID resolution service of IRL resolved standard identification code, in other words, the concrete coding standard of the object identifier can be obtained according to the standard identification code in OID coding. This work was to know standardization organization or enterprise that object identifier belonged to, and then got URL or IP of the specific standards organization or enterprise server. When resolution result was a URL, DNS can be used for further analysis. 


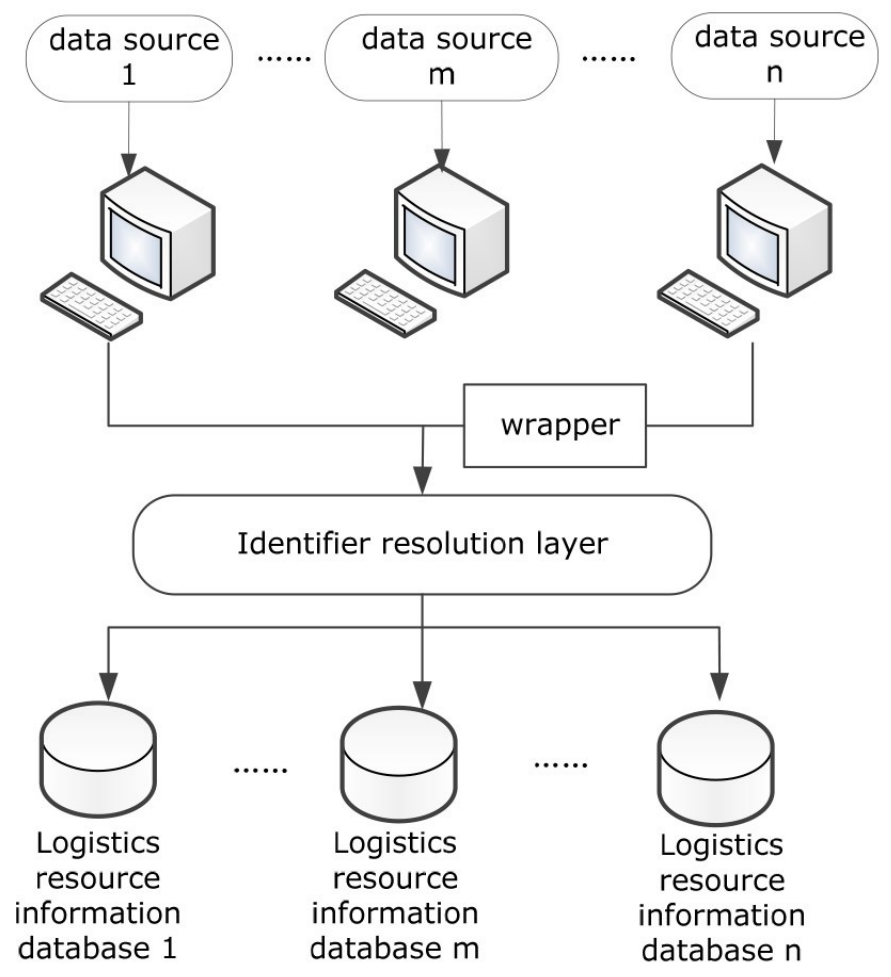

Figure 3. Network Topology of IRL

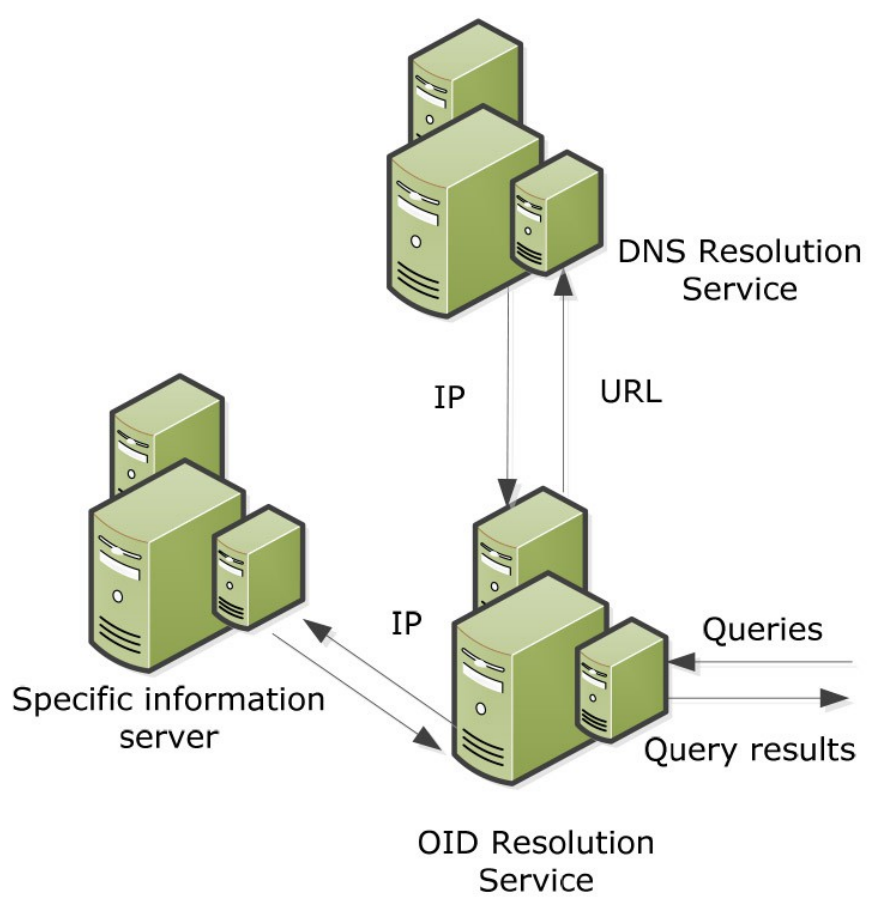

Figure 4. Structure Diagram of IRLIn In 
In order to explain the effect of IRL and how to realize the compatible among different object identifiers, acquisition of logistics resource basic information and location information will be analyzed specifically.

\subsection{Realization of Information Flow}

\section{(1) Acquisition process of logistics resource basic information}

The realization of logistics resources basic information acquisition process was shown in Figure 5.

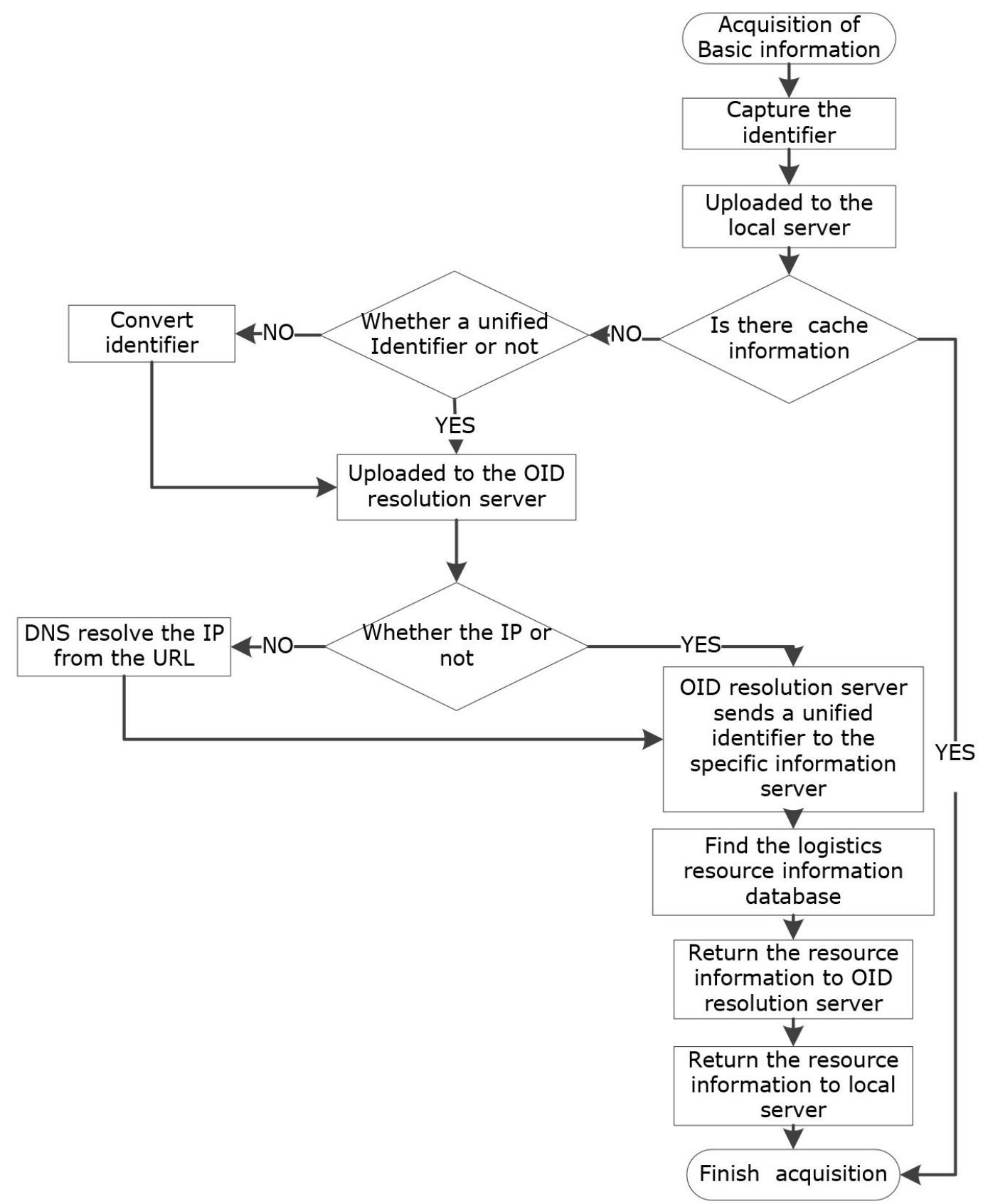

Figure 5. Flow Chart of Accessing to Logistics Resources Basic Information 
1. Read identifier from label and upload it to the local server. When the local server has stored the identifier's corresponding resource basic information, the local server returned basic information directly, otherwise the local server sent the object identifier to the OID resolution server.

2. Two situations may arise when the reader read resource information. (A) It read an OUID contained a standard identification code. (B) It read a resource code. For the case A, the local server sent OUID to OID resolution server, otherwise the resource code needed to be converted into OID encoding format in wrapper, and then be sent to the OID resolution server.

3. OID resolution server can get two results according to OUID based on OID. (A) Organization's URL of the resource basic information. (B) Organization's IP of the resource basic information. When the resolution result was URL, it needed to be resolved by DNS. When the result was IP, OID resolution server directly accessed to the resource basic information in specific server.

4. Specific server provided access path of logistics resource information database, and returned the resource basic information to OID resolution server.

5. OID resolution server returned logistics resource basic information to local server and stored in it. Local server returned information to client. Acquisition of logistics resource basic information has been finished.

The above was acquisition process of logistics resource basic information used OUID, and the process of logistics resource location information still relied on OUID.

\section{(2) Acquisition process of logistics resource location information}

Logistics resource physical location information can be divided into static location information and dynamic location information. It was worth noting that the first record of the resource physical location should be the information recorded by the first resource owner and stored in the information server A (referred to the specific information server in Figure 5 and Figure 6). Logistics resource location information in a certain scope was managed by the information server $A$ within the scope. When the resource flew out of the management of specific information server $A$, server $A$ recorded the resource leave. When the server $B$ of the new location received the information from the resource tag, the server $B$ recorded the resource OUID and new location. Server B sent the information that the resource entered the position B to the original concrete server A (accessing to the original specific information server through addressing OUID). 
The realization of logistics resource location information acquisition process was shown in Figure 6.

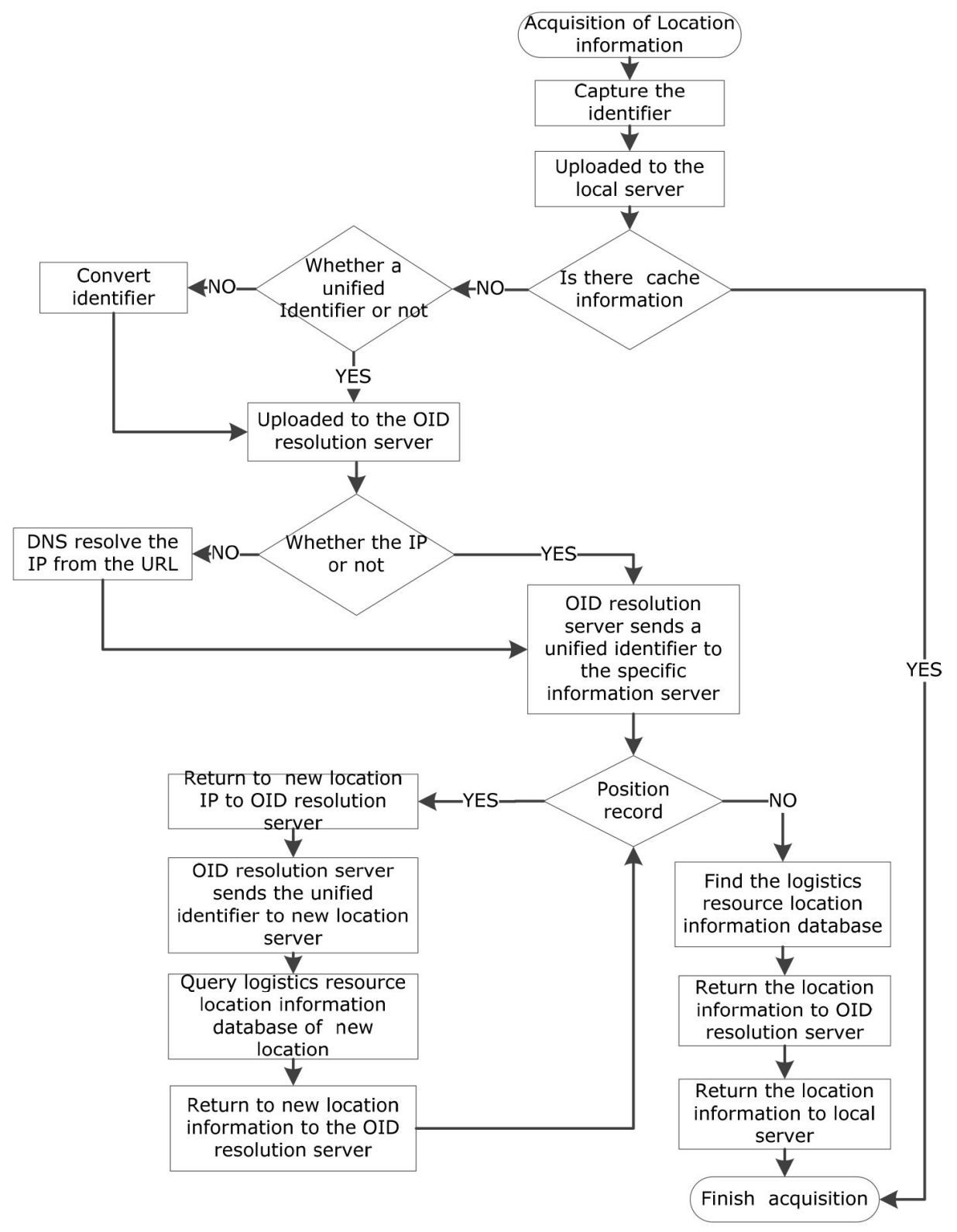

Figure 6. Flow Chart of Accessing to Logistics Resource Location Information

1. Acquisition of static location information. Static logistics resource location information in a certain scope was managed by the location server within the scope. Static logistics resource location information that needed be found can be got by addressing the specific information server that managed the logistics resource through the OUID based on OID. Query process of the specific information server was the same as process of resource basic information. Not be repeated here. 
2. Acquisition of dynamic location information. The resolving process of OUID based on OID remained the same as the process of resource basic information, and then OID resolution server sent OUID to the specific information server. When specific information server had the resource position record, it returned IP of new location to OID resolution server. OID resolution server sent OUID to new location server, and then got new location information. OID resolution sever didn't send OUID to new location servers until there were not position record that hasn't been accessed. Finally, the OID resolution server returned logistics resource location information to the local server, and completed the task of location information query.

From query process of the resource basic information and location information, OUID and IRL can realize the compatible query of logistics resource information on the base of without changing the existing object identifier rules.

\subsection{Application Scenarios}

\section{(1) The application of two-dimensional bar-code}

When company needed to know the delivery information, it can scan the two dimensional barcode to get goods order information, vendor information, product information, etc. However, because of many different kinds of two dimensional bar-code, when mobile phone read twodimensional bar-code, some two-dimensional bar-code information can be obtained only through installing enterprise designated APP on mobile phone. Otherwise, the phone just gets a string of unrecognizable characters. OUID application environment based on OID can be a good solution to this problem.

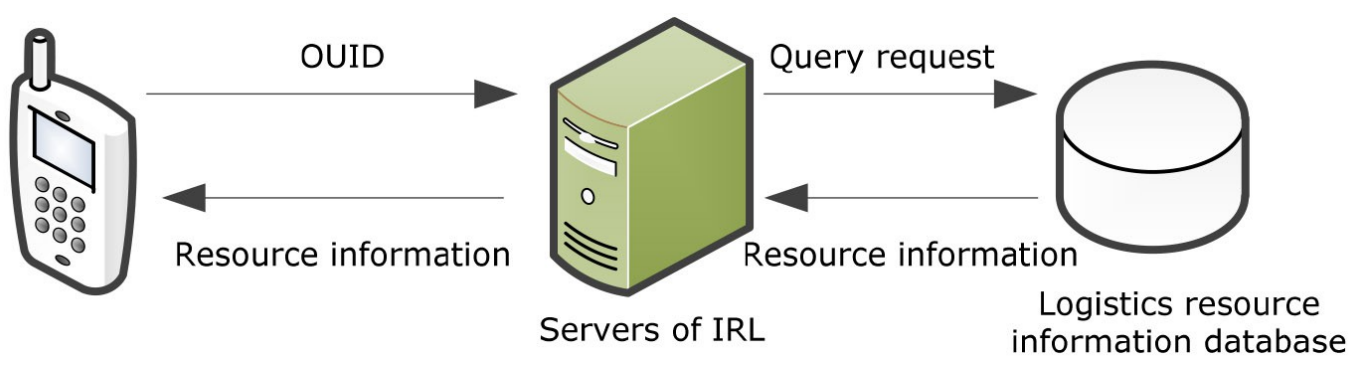

Figure 7. Process of Two Dimensional Bar-code Information Query Using Mobile Phone

When mobile phone read 2-d bar-code, OUID within the bar-code was sent to IRL. Servers of IRL identified the corresponding standard of two dimensional bar-code according to the OID standard identification code. Following that, IRL found the information in accordance with 
information flow mentioned above. At last, using the result of resolution found corresponding logistics resource information database to get the logistics resource information required.

\section{(2) The application of RFID}

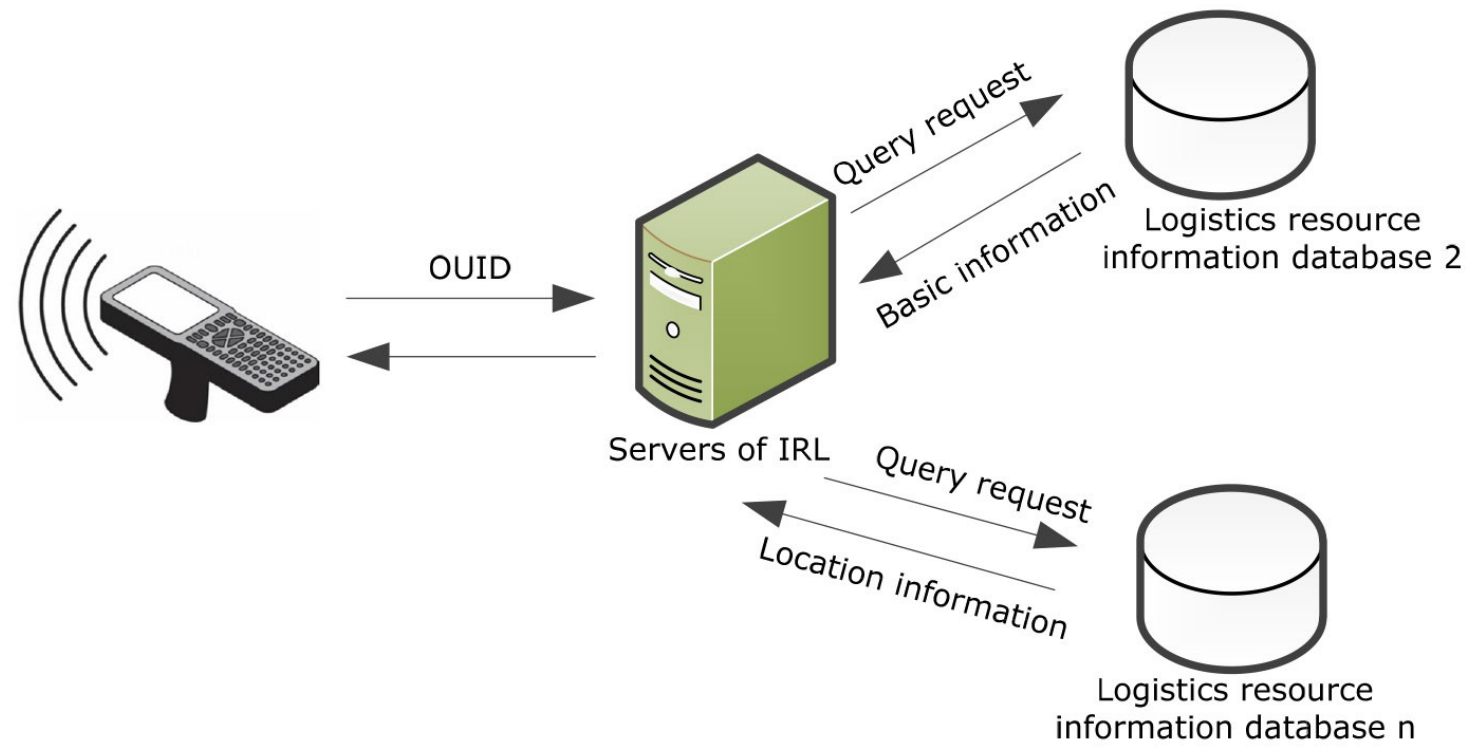

Figure 8. Process of RFID Information Query

When an enterprise wanted to collect the information about the pallets, such as location information of the pallet, vendor information, storage information, etc., it can use the RFID tags on pallets.

Firstly, RFID read-write devices collected OUID within the target scope. Then, they sent OUID to IRL. Because of the strong liquidity of pallets, many servers may have their location information records. So IRL got pallet location information from many specific information servers, besides the pallet basic information. After IRL servers returned the whole pallet information to query terminal, the query task was completed.

From the analysis of application scenarios as you can see, the main function of OUID was to make logistics resource identification information be identified easily by IRL. IRL mainly provided the path for searching information of different object identifier. 


\section{Conclusion}

To ease the influence of non-unified object identifier on logistics resource integration, this study designed the OUID method, and analyzed the OUID application environment by using the computer technologies and standardized method. Specific measures were as follows. (1) Introduce the standard identification code. In order not to change the existing identifier standards, the standard identification code was introduced in object identifier with the help of OID standard. Let object identifier have the OUID structure based on OID. The structure can guarantee object identifier unique in the world, realize compatible query and transformation among multiple object identifiers, and both adapt to the current technology development environment and meet the needs of the logistics resources integration. (2) Construct identifier resolution layer (IRL). The current network structure can't resolve the OUID based on OID, so IRL formed by DNS, OID resolution servers and other servers of standards organizations used to finish resolving OID so as to help address information. This network structure allowed administrators to control data administrator, to realize the traceability of logistics information, classifying information, monitoring and management information, dynamic routing forwarding, etc. On the basis of OUID and IRL, object identifier can confirm logistics resource type, quantity, function, capability and feasible mode of transportation among the various facilities in a certain space through resource static and dynamic location information, except for resource basic information. Moreover, application scenarios of two dimensional bar-code and RFID were list to illustrate the application of OUID method, in order to guide the promotion of OUID.

Overall, OUID proposed both reflected the conversion thought and considered the reality of the Internet information discovery mechanisms. It made up for the lack of location information in conversion mechanism, and avoided to promote new unified identifier standards at the same time. The supplement of the application environment provided an important support to solve the problems of poor communication caused by non-unified object identifier in the process of logistics resource integration. In addition, this paper also had some shortage. For instance, the demand for logistics resource integration OUID was still insufficient in the aspect of data. Therefore, the future research will focus on the above aspect, in order to improve this study.

\section{Acknowledgements}

This study was financially supported by the State Key Program of National Natural Science Foundation of China (Grant No. 71132008). 


\section{References}

CASAGRS. (2010). RFID and the inclusive model for the internet of things. Available at: http://www.grifs-project.eu/data/File/CASAGRAS\%20FinalReport\%20(2).pdf

CERP-IOT. (2009). Internet of things strategic research roadmap. Available at: http://www.grifs-project.eu/data/File/CERP-IoT\%20SRA IoT v11.pdf

GB/T 26231-2010. (2010). Information technology-Open systems interconnection-National numbering system and registration procedures for Object identifier (OID).

Hribernik, K.A., Kramer, C., Hans, C., \& Thoben, K.D. (2010). A Semantic Mediator for Data Integration in Autonomous Logistics Processes. In: K. Popplewell, J. Harding, R. Poler \& R. Chalmeta (Eds.). Enterprise Interoperability IV, 157-167. Springer-Verlag London.

Ilie-Zudor, E., Kemény, Z., van Blommestein, F., Monostori, L., \& van der Meulen, A. (2011). A survey of applications and requirements of unique identification systems and RFID techniques. Computers in Industry, 62(3), 227-252. http://dx.doi.org/10.1016/j.compind.2010.10.004

Jung, E., Choi, Y., Lee, J.S., \& Kim, H.J. (2012). An OID-based identifier framework supporting the interoperability of heterogeneous identifiers. In Advanced Communication Technology (ICACT), 2012 14th International Conference on (304-308). IEEE.

Karakostas, B. (2013). A DNS Architecture for the Internet of Things: A Case Study in Transport Logistics. Procedia Computer Science, 19, 594-601.

http://dx.doi.org/10.1016/j.procs.2013.06.079

Kong, N. (2008). Research on key technology of the resource addressing in the internet of things. PhD. Thesis. Beijing: Graduate University of Chinese Academy of Sciences.

Liu, P., Yan, Z., Tian, Y., Kong, N., Li, X., \& Yan, B. (2014). A Discovery Service for RFID Network Based on Hybrid Architecture. In: J. J. Park, Y. Pan, C. Kim \& Y. Yang (Eds.), Future Information Technology, 849-856. Springer Berlin Heidelberg.

Martinez-Julia, P., \& Skarmeta, A.F. (2013). Beyond the separation of identifier and locator: Building an identity-based overlay network architecture for the Future Internet. Computer Networks, 57, 2280-2300. http://dx.doi.org/10.1016/j.comnet.2012.11.020

Pålsson, H., \& Johansson, O. (2009). Supply chain integration obtained through uniquely labelled goods: a survey of Swedish manufacturing industries. International Journal of Physical Distribution \& Logistics Management, 39(1), 28-46. 
Pang, X.T. (2012). Research on the Resource Addressing Features in the Internet of Things. Information System Engineering, 1, 64-65.

Prajogo, D., \& Olhager, J. (2012). Supply chain integration and performance: The effects of long-term relationships, information technology and sharing, and logistics integration. International Journal of Production Economics, 135(1), 514-522.

http://dx.doi.org/10.1016/j.ijpe.2011.09.001

Romano, P. (2003). Co-ordination and integration mechanisms to manage logistics processes across supply networks. Journal of Purchasing and Supply Management, 9(3), 119-134. http://dx.doi.org/10.1016/S1478-4092(03)00008-6

Shan, L.H., Zhang, Z.Y., Wang X.F., \& Chen, Z.W. (2011). Analysis of Logistics Network and Resources Integration Based on System Theory. Journal of Beijing Jiaotong University (Social Sciences Edition), 10(2), 47-53.

Spleiß, C., \& Kunzmann, G. (2011). A Naming Scheme for Identifiers in a Locator/IdentifierSplit Internet Architecture. In ICN 2011, The Tenth International Conference on Networks (57-62).

Sun, H., Zhang, J.H., Qin, S.W., Tu, Q.W., Wang, X.W., \& Wu, Q.Z. (2013).Research on unified coding system of Internet of things. Application Research of Computer, 30(9), 2707-2710.

Yang, H.R. (2013). Address-based Items Identification and Query Scheme for Internet of Things. Thesis. Guangzhou: South China University of Technology.

Yu, H., \& Yu, X. (2013). A Multi-path Concurrency Object Universal Search (MCOUS) Algorithms based on Code in Internet of Things. Intelligent Computer and Applications, 3(2), 25-31.

Zhang, D. (2012). Internet of things and Object identification system. Internet of things technologies, 2(3), 1-4.

Journal of Industrial Engineering and Management, 2015 (www.jiem.org)

Article's contents are provided on a Attribution-Non Commercial 3.0 Creative commons license. Readers are allowed to copy, distribute and communicate article's contents, provided the author's and Journal of Industrial Engineering and Management's names are included. It must not be used for commercial purposes. To see the complete license contents, please visit http://creativecommons.org/licenses/by-nc/3.0/. 